\title{
Beobachtungen des Cometen 1892 I
}

am Fadenmikrometer des 12 inch. Aequatoreals der Sternwarte in Dresden von Dr. B. von Engelhardt.

\begin{tabular}{|c|c|c|c|c|c|c|c|c|c|c|}
\hline 1892 & M. Z. Dresd. & $\Delta \alpha$ & $\Delta \delta$ & Vgl. & $\alpha$ app. & $\log p .4$ & $\delta$ app. & $\log p \cdot 4$ & Red. ad l. app. & $*$ \\
\hline $\begin{array}{r}\text { ec. } 9 \\
\text { I }\end{array}$ & 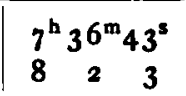 & 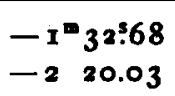 & $\begin{array}{l}-I^{\prime} 19.7 \\
-43^{n} .8\end{array}$ & $\begin{array}{l}21.7 \\
5.5\end{array}$ & $\begin{array}{l}23^{h} 53^{m} 47^{s}: 25 \\
235457.52\end{array}$ & $\begin{array}{l}9.036 \\
9.217\end{array}$ & $\begin{array}{r}+29^{\circ} 42^{\prime} 52^{\prime \prime} .6 \\
+292346.5\end{array}$ & $\begin{array}{l}0.515 \\
0.537\end{array}$ & $\begin{array}{r}+2.24+26.0 \\
+2.24+25.9\end{array}$ & 2 \\
\hline
\end{tabular}

Mittlere Oerter der Vergleichsterne fur 1892.0.

\begin{tabular}{|c|c|c|c|}
\hline * & $\alpha$ & $\delta$ & Autorität \\
\hline $\begin{array}{l}\mathbf{I} \\
\mathbf{2}\end{array}$ & $\begin{array}{l}23^{h} 55^{m} \times 7^{: 69} \\
2357 \quad 15 \cdot 31\end{array}$ & $\begin{array}{r}+29^{\circ} 43^{\prime} 46.3 \\
+292753.4\end{array}$ & $\begin{array}{l}\text { BB. VI }+29: 5043 \\
W_{2} 23^{h} \text { I } 183\end{array}$ \\
\hline
\end{tabular}

Bemerkungen.

Dec. 9. Luft nebelig. Comet schwach, Durchmesser $I^{\prime} \pm$, unfassbare Conturen, granulirtes Aussehen, welches wahrscheinlich von sehr kleinen bedeckten Sternen herruhrt; Verdichtung, Kern, auf welchen eingestellt wurde; es ist aber nicht unmöglich, dass derselbe nicht der Cometenkern, sondern ein Sternchen ist. - Dec. $\mathrm{r}$ r. Luft schlecht. Comet sehr schwach, rund, etwa $1 / 2^{\prime}$ Durchmesser, kein Kern; mehrere kleine Lichtpunkte, welche wahrscheinlich kleine Sterne sind, blitzen bisweilen auf und erschweren die Pointirungen.

Dresden 1892 December.

Dr. B. von Engelhardt.

Beobachtungen des Cometen 1892 III (Holmes) am $18 z$. Refractor der Kais. Universitätssternwarte in Strassburg.

\begin{tabular}{|c|c|c|c|c|c|c|c|c|c|c|}
\hline 1893 & M.Z.Strassb. & $\Delta x$ & $\Delta \delta$ & Vgl. & $\alpha$ app. & $\log p . \Delta$ & $\delta$ app. & $\log p .4$ & Red. ad l. app. & 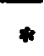 \\
\hline Jan. 5 & $\begin{array}{lrl}6^{\mathrm{h}} & 19^{\mathrm{m}} & 22^{\mathrm{s}} \\
6 & 3 & 26\end{array}$ & $\begin{array}{l}+0^{=1} 10^{5} .32 \\
-026.08\end{array}$ & $\begin{array}{l}-25.8 \\
+331.5\end{array}$ & $\mid \begin{array}{c}10.7 \\
12.12\end{array}$ & 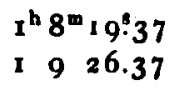 & $\begin{array}{l}8.427 \\
6.316\end{array}$ & $\begin{array}{l}+33^{\circ} 49^{\circ} 8.6 \\
+334754.4\end{array}$ & $\begin{array}{l}0.348 \\
0.348\end{array}$ & $\begin{array}{l}-0.77+8.5 \\
-0.77+8.5\end{array}$ & I \\
\hline
\end{tabular}

Mittlere Oerter der Vergleichsterne für 1893.0.

\begin{tabular}{|c|c|c|c|}
\hline$\bullet$ & $\alpha$ & $\delta$ & Autorităt \\
\hline $\begin{array}{l}\mathbf{I} \\
\mathbf{2}\end{array}$ & $\begin{array}{r}1^{\mathrm{h}} 8^{\mathrm{m}} 9: 82 \\
1953.22\end{array}$ & $\begin{array}{rr}+33^{\circ} 51^{\circ} & 5.9 \\
+3344 & 14.4\end{array}$ & $\begin{array}{l}\text { Leid. AG. Z. } 343,347 \\
\text { Leid. AG. Z. } 343,393\end{array}$ \\
\hline
\end{tabular}

Jan. 5. Comet ein blasser Lichtfleck von 2' Ausdehnung, der nur mit grosser Anstrengung zu erkennen ist, bei etwas dunstiger Luft. - Jan. 6. Ein Stern $15^{\mathrm{m}}$, welcher $2^{\mathrm{s}}$ voraufgeht, stört die Beobachtung. Bei nebeliger Luft Beobachtung ausserordentlich schwierig.

Strassburg 1893 Jan. 7.

H. Kobold.

Sur la solution d'équations normales.

Par $H$. Geelmuyden.

D'après le procédé ordinaire pour la solution des équations qu'on obtient par la méthode des moindres carrées, tel qu'on le trouve p. e. amplement exposé dans le livre d'Oppolzer , Lehrbuch der Bahnbestimmung der Planeten und Cometenk, on substitue la première des inconnues, exprimée par les autres, dans toutes les équations suivantes, et ainsi de suite. Lorsqu'on peut se contenter du degré de précision que donne une règle-a-calcul, il est plus naturel de procéder d'une autre manière qui abrège le calcul. La règle-à-calcul ne donne en général plus de trois chiffres, mais il est facile d'en étendre l'exactitude par diverses artifices; ainsi pour une division on peut calculer de tête le premier ou les deux premiers chiffres du quotient et puis prendre le reste sur la règle; si le premier chiffre valant du quotient est grand, on peut prendre son complement a une unité décade en substituant au dividende la différence entre le diviseur et le dividende; aussi pour une multiplication on peut souvent se servir du complément.

Le procédé le plus naturel est alors de diviser chacune des équations normales par la valeur numérique 
du coefficient de l'inconnue qu'on veut éliminer et qu'on peut choisir à volonté, et puis d'effectuer l'élimination par simple soustraction ou addition de deux équations qu'on peut aussi choisir à volonté, pourvu qu'on fasse le nombre nécessaire de combinaisons indépendantes; ainsi de suite jusqu'à ce qu'on finisse par une équation avec une seule inconnue. (S'il y a des équations qui ne contiennent pas toutes les inconnues, on peut de cette manière éviter l'inconvénient d'introduire une inconnue dans une équation où elle ne se trouve pas auparavant). Alors commence la partie des opérations qu'on peut appeler le calcul rétrograde, c'est-à-dire la substitution de la derniere inconnue dans l'une des équations qui contient l'avant-dernière avec le coefficient +1 ou -1 , et ainsi de suite; naturellement on choisit toujours l'équation où les inconnues déjà déterminées ont les plus faibles coefficients.

Ordinairement il s'agit de déterminer non seulement la valeur des inconnues, mais aussi leur poids relatif. Il est facile de mener le calcul vers ce but. Soient données $\boldsymbol{n}$ équations aux $\boldsymbol{n}$ inconnues $x, y$. . :

$$
\begin{aligned}
& a_{1} x+a_{2} y+a_{3} z+\cdots=A \\
& b_{1} x+b_{2} y+b_{3} z+\cdots \cdot=B \\
& f_{1} x+f_{2} y+f_{3} z+\cdots=F
\end{aligned}
$$

Si l'on commence par l'élimination de $x$, en divisant chaque équation par la valeur numérique de $a_{1}, b_{1}, \ldots$. $f_{1}$, on obtient, après la combinaison de deux et deux équations, un nouveau système de $n-I$ équations :

$$
\begin{aligned}
& g_{2} y+g_{3} z+\cdots \cdot=G \\
& h_{2} y+h_{3} z+\cdots \cdot=H
\end{aligned}
$$

où les quantités $G, H, \ldots$ sont des fonctions linéaires de deux des quantités $A, B$, . (ou d'une seule, si une ou plusieurs des équations ( 1 ) ne contiennent pas $x$ ); après une nouvelle élimination on obtient $x-2$ equations dont les termes à droite sont des fonctions linéaires de trois ou quatre des quantités $A, B, \cdots \cdot$, et ainsi de suite, jusqu'à ce que la dernière des inconnues se trouve exprimée par toutes ces quantités. Or en se rappelant que, si les équations (I) sont des équations normales, le poids de $\boldsymbol{x}$, $y, z$. . est exprimé par le réciproque des coefficients $\alpha_{1}, \beta_{2}, \gamma_{3} \ldots$ dans les équations:

$$
\begin{aligned}
& x=\alpha_{1} A+\alpha_{2} B+\alpha_{3} C+\cdots+\alpha_{n} F \\
& y=\beta_{1} A+\beta_{2} B+\beta_{3} C+\cdots+\beta_{n} F \\
& z=\gamma_{1} A+\gamma_{2} B+\gamma_{3} C+\cdots+\gamma_{n} F
\end{aligned}
$$

on voit qu'il suffît de conserver les symboles $A, B$, . . pendant tout le calcul et de ne substituer leurs valeurs que lorsqu'on en a besoin. Comme un contrôle partiel on peut aussi faire le calcul des termes à droite doublement, c'est-à-dire d'opérer non seulement avec les fonctions linéaires de $A, B$, . ., mais aussi avec le chiffre équivalent qu'on obtient en employant leurs valeurs dès le commencement. Dans le calcul rétrograde on peut omettre successivement celle des quantités $A, B$, . . dont le co. efficient a déjà servi à déterminer le poids d'une inconnue; mais en les conservant toutes jusqu'd la fin, on obtient un contrôle final pour la détermination des poids, aussitôt qu'on ait contrôlé la détermination des inconnues par la substitution de leurs valeurs dans les équations ( $\mathrm{r}$ ).

Si l'on cherche les expressions générales des coefficients $\alpha_{1}, \beta_{2}, \ldots$ en fonctions des coefficients qu'on a trouvé par le calcul direct, on trouve une expression trèssimple pour le dernier et aussi pour l'avant-dernier, mais pour les autres la complication va en croissant avec le nombre des inconnues. Pour le calcul on n'en a pas besoin.

Christiania 1892 nov. I 2.

H. Geelmuyden.

\section{Ueber eine plötzliche Aenderung im Aussehen des Cometen 1892 III (Holmes).}

Die Centralstelle erhielt am 16. d. M. Abends 9 Uhr folgendes Telegramm aus Wien:

,Comet Holmes soeben 7 Uhr gleicht Fixstern achter Grösse mit Nebelhülle von 20 Bogensecunden Durchmesser. Palisa.k

Diese merkwurdige Beobachtung wurde sofort durch Telegramm weiter gegeben.

Gleich darnach klärte sich hier der Himmel für kurze Zeit auf und Prof. Lamp konnte die Beobachtung von Dr. Palisa bestätigen, ohne dass indessen eine Positionsbestimmung erlangt werden konnte.

Kiel 1893 Jan. 17.

Kr.

\section{In halt:}

Zu Nr. 3145. S. von Glasenapp. Bestimmung der Bahn des Doppelsterns $\mathbf{2}$ 2. 1. - S. von Glasenapp. Neue Bestimmung der Bahn des Doppelsterns 85 Pegasi $=\beta$ 733. 3. - G. Witt. Beobachtungen von Cometen. 7. - B. von Engclhardt. Beobachtungen des Cometen 1892 I. 13. - H. Kobold. Beobachtungen des Cometen 1892 III (Holmes). 13. - H. Geelmuyden. Sur la solution d'équations normales. 13. - Ueber eine plötzliche Aenderung im Aussehen des Cometen 1892 III (Holmes). I5. 